\title{
Preferential survival of mice expressing the Qa-2 antigen
}

\author{
C. M. Warner, P. Panda, C. D. Almquist and Y. Xu \\ Department of Biology, 414 Mugar Hall, Northeastern University, Boston, MA 02115, USA
}

\begin{abstract}
The Ped gene, a gene that influences the rate of embryonic cleavage division, birth weight, litter size and weaning weight, is at least partially encoded by gene(s) that specify the Qa-2 antigen. Two congenic strains of mice, B6.KI (Qa-2 negative) and B6.K2 (Qa-2 positive), which differ only at the $Q$ region of the mouse major histocompatibility complex (MHC), were tested for the effect of the presence or absence of Qa-2 antigen on litter size, duration of gestation and embryo survival. It was confirmed that B6.KI (Qa-2 negative) mice have smaller litters than do B6.K2 (Qa-2 positive) mice. In addition, the duration of gestation for the B6.K1 mice was found to be longer than the duration of gestation for the B6.K2 mice. Finally, a comparison of the relative survival of Qa-2-positive and Qa-2-negative mice in a single uterine environment showed the preferential survival of mice expressing the Qa-2 antigen. Thus, the presence of Qa-2 antigen appears to be advantageous for reproductive success.
\end{abstract}

\section{Introduction}

Embryo survival is mediated by environmental and genetic components. In mice, at least some of the genes that influence embryo survival are located in the major histocompatibility complex (MHC). One gene of particular interest in the MHC is the Ped gene, which controls the rate of cleavage division of preimplantation mouse embryos as well as embryo survival (reviewed in Warner et al., 1988, 1991).

The Ped gene has been mapped to the $\mathrm{Q}$ region of the mouse MHC (Warner et al., 1991), a region that contains ten known class I genes, Q1-Q10 (reviewed in Flaherty et al., 1990; Stroynowski, 1990; Morse et al., 1991). However, only three protein products encoded by the $Q$ region have been identified: $\mathrm{Qb}-1$, encoded by the $\mathrm{Q} 4$ gene; $\mathrm{Q} a-2$, encoded by the four very similar genes Q6, Q7, Q8, and Q9; and Q10, encoded by the Q10 gene. Of these genes, the transcription (Jin et al., 1992) and expression (Warner et al., 1987; Tian et al., 1992) of the Qa-2 antigen encoding genes have been studied in some detail in preimplantation mouse embryos. It has been hypothesized that the Qa-2 antigen is the product of the Ped gene (Wamer $e$ al., 1987; Tian et al., 1992). Recent experiments using antisense oligonucleotides to Qa-2 antigen mRNA have shown directly that the $\mathrm{Q7}$ gene or $\mathrm{Q} 9$ gene or both, each of which encodes the Qa-2 antigen, are at least partially responsible for the Ped gene phenotype of preimplantation mouse embryos $(X u$ and Warner, 1992; Xu et al., 1993). The studies reported here were undertaken to assess the role of Qa-2 antigen in embryo survival. Two congenic mouse strains, B6.KI and B6.K2, which differ only at the $\mathrm{Q}$ region of the mouse MHC, were chosen for these studies. Data are reported in which the survival of mice expressing the Qa-2 antigen is compared with the survival of those that do not express the Qa-2 antigen.

\section{Materials and Methods}

\section{Animals}

The B6.K1 (Qa-2 negative) and B6.K2 (Qa-2 positive) congenic strains of mice, originally obtained from L. Flaherty (Wadsworth Center, Albany, NY) in 1985, were bred and maintained in our laboratory. These mice differ only at the $Q$ region of the mouse MHC (Flaherty, 1976). The B6.K1 mice have a slower embryonic cleavage rate (Ped slow), a lower birth weight, a smaller litter size, and a lower weaning weight than the B6.K2 mice (Warner et al., 1988, 1991). The (B6.K1 $\times$ B6.K2) $F_{1}$ mice and $(B 6 . K 1 \times B 6 . K 2)$ male $\times$ B6.K1 female backcross mice were produced in our laboratory. The mice were housed in a light controlled room (lights on 04:00-18:00 h standard time) with food and water available ad libitum.

\section{Time of gestation}

Five breeding cages for each strain of mice were set up containing one male and three to five females so that the duration of gestation of pups resulting from homozygous matings of B6.K1 male $\times$ B6.K1 female and B6.K2 male $\times$ B6.K2 female mice could be determined. The female mice were checked at 08:00 $\mathrm{h}$ each morning for the presence of a vaginal plug and mice with a plug were removed and placed in individual cages. The pregnant mice were monitored for birth of litters at 08:00, $12: 00$, and $17: 00 \mathrm{~h}$ starting at 18 days $(432 \mathrm{~h})$ after detection of the plug. The time of birth and number of pups per litter were recorded. The gestation time was calculated as the number of hours after the detection of the vaginal plug.

\section{Embryo survival in backcross mice}

A backcross was set up in which (B6.K1 $\times$ B6.K2) male mice were mated with $\mathrm{B} 6 . \mathrm{K} 1$ female mice to assess the relative survival of Qa-2-positive mice and Qa-2-negative mice in a 
Table 1. Mean gestation time of B6.KI and B6.K2 mice

\begin{tabular}{|c|c|c|c|c|c|}
\hline Strain & $\begin{array}{l}\text { Number of } \\
\text { litters }\end{array}$ & $\begin{array}{l}\text { Mean number pups/ } \\
\text { litter } \pm \text { SEM }\end{array}$ & $P$ & $\begin{array}{c}\text { Duration of } \\
\text { gestation } \pm \text { SEM } \\
\text { (h) }\end{array}$ & $P$ \\
\hline B6.K1 & 10 & $5.4 \pm 0.3$ & & $490 \pm 3.9$ & \\
\hline B6.K2 & 24 & $8.1 \pm 1.3$ & $<0.001$ & $476 \pm 2.4$ & $<0.01$ \\
\hline
\end{tabular}

single uterine environment. Only those litters in which all the pups were live-born and survived for two months were used in the analysis. At two months of age, the Qa-2 antigen phenotype of the offspring from the cross was determined by immunofluorescence (Janick-Buckner et al., 1991). Briefly, the spleen was excised from each mouse, the lymphocytes isolated using a Ficoll-Hypaque density gradient, and the cells were adjusted to a concentration of $5 \times 10^{6} \mathrm{ml}^{-1}$. The lymphocytes were stained using an anti-Qa-2 monoclonal antibody, 1-12-1 (Sharrow et al., 1989), obtained from L. Flaherty, as the first antibody and by using a fluorescein isothiocyanate (FITC) conjugated goat anti-mouse Fc antibody (ICN Biomedicals, Irvine, $\mathrm{CA}$ ) as the second antibody. After the assay, 3\% formaldehyde was added to each tube and the cells were stored overnight at $4^{\circ} \mathrm{C}$. The results were read the next day using a FACScan flow cytometer. Details of the FACScan analysis and validation of its accuracy have been published by Armstrong and Warner (1992).

\section{Statistical analyses}

The data on the mean gestation time were analysed by Student's $t$ test. The data on preferential survival of Qa-2positive offspring were analysed by $\chi^{2}$ analysis.

\section{Results}

In the first set of experiments the mean gestation time of B6.KI mice was compared with that of B6.K2 mice. The results (Table 1 ) show that the duration of gestation is longer for the B6.K1 than for the B6.K2 strain. However, even though the B6.K1 mice remain in utero for longer, they are still smaller at birth than their B6.K2 counterparts (Wamer et al., 1991). The data in Table 1 also confirm previously reported data (Warner et al., 1991) that the mean number of pups is higher for the B6.K2 strain than for the B6.K1 strain.

In the second set of experiments, the relative survival of Qa-2-positive and Qa-2-negative mice in a single uterine environment was assessed. If $\mathrm{Q} a-2$ antigen expression is not linked to embryo survival, the ratio of Qa-2-positive:Qa-2negative mice should be $50: 50$ in the (B6.KI $\times$ B6.K2) male $\times$ B6.K1 female backcross. All of the offspring from the backcross were analysed for $\mathrm{Qa}-2$ antigen expression by immunofluorescence. A typical profile of the Qa-2-positive (B6.K2) and Qa-2-negative (B6.K1) controls, and the profile from an unknown mouse are shown (Fig. 1). It is easy to see that the unknown mouse in this case is Qa-2-positive. Thus, this
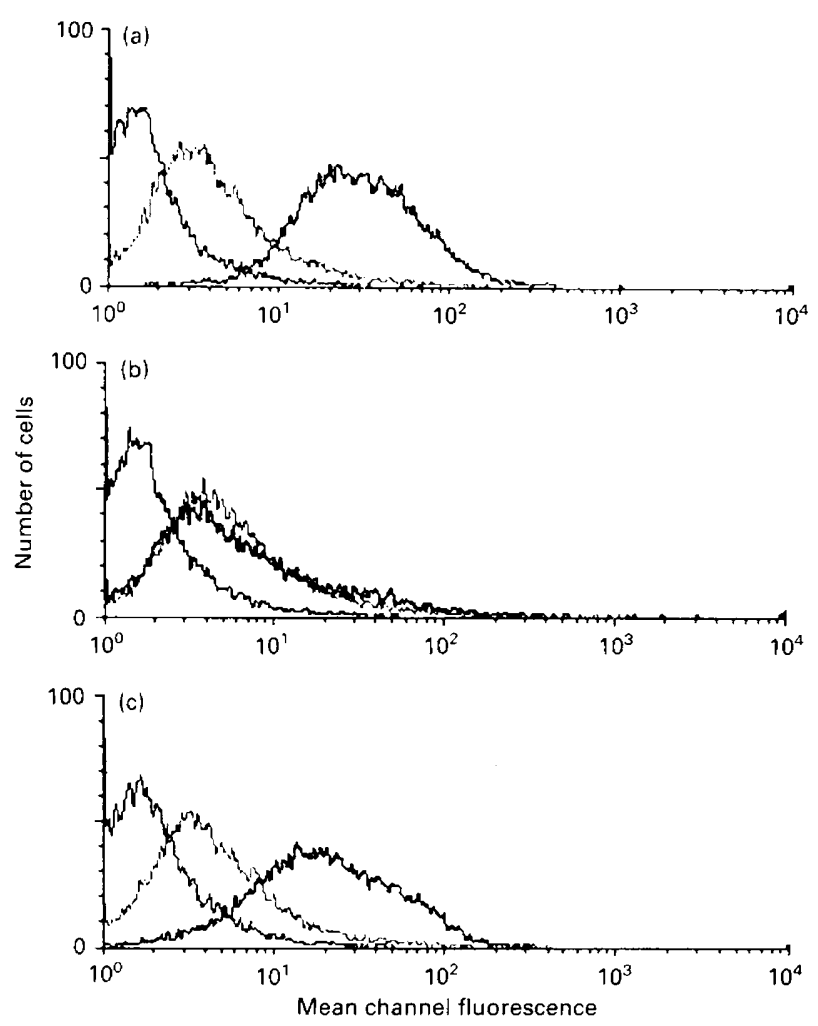

Fig. 1. Detection of Qa-2 antigen by immunofluorescence. Lymphocytes were isolated and tested for Qa-2 antigen expression by using a FACScan flow cytometer, as described in the text. (a) B6.K2 (Qa-2-positive); (b) B6.KI (Qa-2-negative); (c) unknown mouse (Qa-2-positive). In (a) the curve on the left is autofluorescence, the curve in the middle is background nonspecific binding of the fluorescein isothiocyanate (FITC)-labelled second antibody alone, and the curve on the right is specific binding of the FITC-labelled second antibody to the anti-Qa-2 first antibody.

method has provided an excellent method to distinguish Qa-2positive from Qa-2-negative mice. The results of the analysis of Qa-2 antigen expression by 62 offspring from the backcross show greatly enhanced survival of Qa-2-positive compared with Qa-2-negative offspring from the backcross (Table 2).

\section{Discussion}

The results reported in this paper show that mice expressing the Qa-2 antigen show preferential survival compared with mice that do not. By setting up the backcross experiment, we 
Table 2. Chi-square analysis of Qa-2 phenotype of (B6.KI $\times$ B6.K2) male $\times$ B6.KI female backcross mice

\begin{tabular}{lccc}
\hline & \multicolumn{2}{c}{ Number of offspring } \\
Qa-2 phenotype & Observed & Expected & $P^{a}$ \\
\hline Positive & 45 & 31 & $<0.01$ \\
Negative & 17 & 31 & $<0.01$ \\
\hline
\end{tabular}

${ }^{2}$ The hypothesis tested was that $Q \mathrm{a}-2$ antigen is not linked to embryo survival. This would result in $50 \%$ of the offspring as Qa-2 positive and $50 \%$ of the offspring as Qa-2 negative. The observed values were different from the expected values at $p<0.01$. The hypothesis is therefore rejected and the conclusion is that there is preferential survival of embryos which express Qa-2 antigen.

investigated development of both Qa-2-positive and Qa-2negative mice in the same uterine environment. The results obtained showed markedly enhanced survival of Qa-2-positive mice compared with Qa-2-negative mice. It will be interesting to determine exactly when in gestation the enhanced survival of the Qa-2-positive embryos occurs: it could occur during the preimplantation period, at implantation, during gestation, at birth, neonatally, or during the first two months after birth.

The mechanisms by which the presence of Qa-2 antigen enhances embryonic survival remain to be determined. The Qa-2 antigen is an MHC class I protein and therefore is considered to have the same biochemical structure as other members of this family (reviewed in Flaherty et al., 1990; Stroynowski, 1990; Morse et al., 1991). The X-ray crystallographic structure of a mouse MHC class I protein has recently been reported (Fremont et al, 1992) and the mouse class I protein structure is very similar to the previously reported structure of a human class I protein. The hallmark feature of the class I proteins is a binding groove for an approximately nine amino acid long peptide in a pocket formed by the $\alpha 1$ and $\alpha 2$ domains. The nature of the peptide(s) bound by Qa-2 antigen is unknown. It is possible that binding of a particular peptide will be a mediator of enhanced embryo survival.

The other part of the Qa-2 molecule of particular biochemical interest is the membrane-binding portion of the molecule. Qa-2 antigens may be bound to the cell membrane either by a transmembrane domain or by a glycosylphosphatidylinositol (GPI) linkage. We have recently shown that all of the Qa-2 antigen on preimplantation mouse embryos is sensitive to cleavage by phospholipase $C$ and is therefore bound to the embryonic cell surface by GPI linkage (Tian et al., 1992). It is possible that the GPI linkage of the Qa-2 antigen to the cell membrane mediates signals to the embryo from its environ- ment. Perhaps those embryos without Qa-2 antigen cannot receive the necessary signals to enhance their probability of survival. Future studies in which transgenic mice are created with genetically engineered $\mathrm{Qa}-2$ molecules may shed further light on the molecular mechanisms by which the presence of Qa-2 antigen enhances embryo survival.

This work was supported by NIH grant HD 13748 .

\section{References}

Armstrong MJ and Warner CM (1992) Expression of phosphatidylinositoldependent phospholipase $\mathrm{C}$ sensitive $\mathrm{Qa}-2$ antigen is increased on peripheral blood lymphocytes of aging mice Growth, Development and Aging 56 $225-236$

Flaherty L (1976) The Tla region of the mouse: identification of a new serologically defined locus, Qa-2 Immunogenetics 3 533-539

Flaherty L, Elliot E, Tine JA, Walsh AC and Waters JB (1990) Immunogenetics of the $\mathrm{Q}$ and TL regions of the mouse Critical Reviews of Immunology 10 131-175

Fremont DH, Matsumura M, Stura EA, Peterson PA and Wilson IA (1992) Crystal structures of two viral peptides in complex with murine MHC class I $\mathrm{H}-2 \mathrm{~K}^{\mathrm{b}}$ Science 257 919-926

Janick-Buckner D, Briggs CJ, Meyer TE, Harvey N and Warner CM (1991) Major histocompatibility complex antigen expression on lymphocytes from aging strain A mice Growth Development and Aging 55 53-62

Jin P, Meyer TE and Warner CM (1992) Control of embryo growth by the Ped gene: use of reverse transcriptase polymerase chain reaction (RT-PCR) to measure mRNA in preimplantation embryos Assisted Reproductive Technology Andrology 3 377-383

Morse RY, Watts W, Gelber C and Goodenow RS (1991) The Qa region genes and antigens of the murine major histocompatibility complex. In Immunogenetics of the Major Histocompatibility Complex, pp 155-176 Eds R. Strinivastava and B Ram. VCH Publishers, New York

Sharrow SO, Arn JC, Stroynowski I, Hood L and Sachs D (1989) Epitope clusters of Qa-2 antigens defined by a panel of new monoclonal antibodies journal of Immunology 142 3495-3502

Stroynowski I (1990) Molecules related to class-I major histocompatibility complex antigens Annual Reviews of Immunology 8 501-530

Tian Z, Xu Y and Warner CM (1992) Removal of Qa-2 antigen alters the Ped gene phenotype of preimplantation mouse embryos Biology of Reproduction $47271-276$

Warner CM, Gollnick SO, Flaherty L and Goldbard SB (1987) Analysis of Qa-2 antigen expression by preimplantation mouse embryos: possible relationship to the Ped gene product Biology of Reproduction 36 611-616

Warner CM, Brownell MS and Ewoldsen MA (1988) Why aren't embryos immunologically rejected by their mothers? Biology of Reproduction $38 \quad 17-29$

Warner CM, Brownell MS and Rothschild MF (1991) Analysis of litter size and weight in mice differing in Ped gene phenotype and $\mathrm{Q}$ region of the $\mathrm{H}-2$ complex Journal of Reproductive Immunology 19 303-313

$\mathrm{Xu} \mathrm{Y}_{\mathrm{u}}$ and Warner CM (1992) Effect of antisense oligonucleotides to major histocompatibility complex (MHC) mRNA on preimplantation embryo development Assisted Reproductive Technology Andrology 3 219-223

Xu Y, Jin P and Warner CM (1993) Modulation of preimplantation embryonic development by antisense oligonucleotides to MHC genes Biology of Reproduction 48 1042-1046 